\title{
Research of properties on graphite conductive slag in asphalt concrete
}

\author{
Jian Sun ${ }^{1}$, Andrii Bieliatynskyi ${ }^{2,3}$, Kateryna Krayushkina ${ }^{2}$, Oleksandra Akmaldinova, ${ }^{2, *}$ \\ ${ }^{1}$ Kharkov National Automobile highway University, 25, str. Yaroslav the Wise, 61002 Kharkov, \\ Ukraine \\ ${ }^{2}$ National Aviation University, 1, Lubomyra Gusara Ave., 03058 Kyiv, Ukraine \\ ${ }^{3}$ North Minzu University, 204, Nort-Wenchang St. Xixia District, Yinchuan, Ningxia, People's \\ Republic of China
}

\begin{abstract}
Conductive asphalt concrete can eliminate snow and icing on road areas, and is a research hot topic for intelligent snow removal. By adding graphite, the electrical resistivity of asphalt concrete can be reduced to achieve the purpose of electrical conductivity. However, the aggregate electrical resistance in asphalt concrete is very large and does not play a conductive role. It is a new technical idea to use steel slag with good electrical conductivity as aggregate to mix graphite powder with good electrical conductivity to prepare steel slag graphite conductive asphalt concrete (SGCAC). In this paper, the effects of steel slag and graphite on the conductive properties of steel slag graphite conductive asphalt concrete are separately explained, and then the conductive mechanism and electrical resistance stability of the conductive slag and graphite are studied.
\end{abstract}

\section{Introduction}

After snowfall in winter in high and cold areas, roads are prone to snow or icing, which brings huge safety risks to transportation, affects people's safe travel, and even causes the entire transportation network to be paralyzed. With the development and progress of science and technology, human snow removal methods have also made great progress. At present, the most commonly used snow removal method at home and abroad is to spray deicing salt, but a large amount of spraying deicing salt will cause erosion of the road surface and greatly reduce its service life. Therefore, people began to explore automatic snow removal methods, by heating the road surface and making it warm, to achieve the purpose of melting snow and icing. At present, the research on automatic snow removal methods mainly includes ground pipe heating method, new solar heat pipe method, infrared lamp heating method, and conductive concrete. As the conductive asphalt concrete itself has the ability to conduct electricity, there is no need to bury electric heating elements, and it will not adversely affect the pavement structure, so it has become a research hot topic for

\footnotetext{
*Corresponding author: 1586118851@qq.com
} 
intelligent snow removal.

In the 1930s, scientists began conducting research on conductive asphalt concrete. In 1971, the Soviet Union achieved an ideal deicing effect by mixing $22 \%$ by volume of graphite in conductive asphalt as a conductive material [1].At present, the research of conductive asphalt concrete mainly forms conductive network in concrete by adding conductive phase material to cementitious materials. However, the proportion of cementitious materials in the concrete is only about $1 / 3$, while the aggregates that account for about $2 / 3$ do not play a conductive role, but instead cut off some conductive networks. In order to solve this problem, it is necessary to find a material that has both good mechanical properties and excellent electrical conductivity.

\section{Effect of steel slag using}

With the continuous development of the steel industry, a large amount of steel slag is treated as waste. Not only has it caused tremendous pressure on the production and development of iron and steel enterprises, but it has also occupied land and polluted the environment. Therefore, many countries have invested a lot of labor, material resources and financial resources to study the problem of steel slag utilization. Initially, steel slag was used as the bottom and base course materials in road engineering. In recent years, steel slag has been used as an aggregate and incorporated into asphalt concrete as a surface course material.

Steel slag is a by-product formed during the steelmaking process to remove impurities in the molten iron. It is a compressed pore structure rich in iron elements, in which the FeO content is high and it contains a large amount of metallic iron. Since both the elemental iron and the divalent iron in the steel slag are good conductors, it was found by measuring the volume resistivity that the steel slag has excellent electrical conductivity. During the electrification process, electrons can be transferred between different valence irons rapidly, thereby realizing the movement of electrons inside the asphalt concrete aggregate [2].

Lv Linnv et al. found through experiments that the steel slag coarse aggregates were connected to each other in the asphalt mixture to form a conductive path. The steel slag fine aggregate is composed of asphalt; fiber stabilizer, mineral powder, and a small amount of fine aggregate to form the asphalt mastic fat to fill the gap of the coarse aggregate skeleton, which further improves the conductive path. With the increase of the amount of steel slag, the conductive path is perfect.

Using steel slag as an aggregate in asphalt concrete is conducive to improving the electrical conductivity of asphalt concrete, and plays an important role in achieving snow melting and deicing on the pavement, safety monitoring and intelligent management.

\section{Effect of graphite using}

Graphite is an isomer of elemental carbon. It has good electrical and thermal conductivity, stable chemical properties and excellent durability. In addition, graphite powder is cheaper than carbon fiber and has a good dispersion effect in asphalt concrete. Mo Liantong et al. believed that the particle size, oil absorption value, $\mathrm{pH}$ value and specific surface area of graphite have a greater impact on the conductivity. As a conductive material, graphite has high structure, large specific surface area and good surface chemistry [4,5]. Therefore, the conductivity formula of asphalt concrete is put forward: 


$$
\delta=\frac{3\left(\frac{3 L}{d}-2\right)\left(V_{f}-\frac{4 d}{3 L}\right)}{R_{0} L}
$$

Where $\delta$ - the electrical conductivity of the asphalt concrete;

$\mathrm{L}$ - the side length of the cube specimen;

$\mathrm{d}$ - the diameter of graphite particles;

$\mathrm{V}_{\mathrm{f}}$ - the volume fraction of graphite;

$\mathrm{R}_{0}$ - the contact electrical resistance.

It can be seen from the formula that the particle size of graphite, the content of graphite, and the contact electrical resistance are important factors that determine the resistivity of conductive asphalt concrete. In the case where the graphite volume fraction is constant, the smaller the graphite particle size, the larger the specific surface area, the higher the graphite dispersion, the easier it is to form a conductive network and the better the conductive performance

The influence of graphite content on the conductivity of asphalt concrete can be divided into three stages: insulation stage, transition stage and conduction stage. In SGCAC, when the graphite content is very low, graphite and asphalt form a two-phase composite conductive material with high electrical resistance, which cuts off part of the conductive network between the steel slag. As the amount of graphite increases, the electrical resistance of the two-phase composite conductive material formed by graphite and asphalt decreases, and the conductive network between the steel slags is connected. The higher the amount of graphite, the more complete the conductive path formed.

In general, graphite can effectively reduce the electrical resistance value of asphalt concrete, and the greater the amount of graphite, the more significant the effect. Due to graphite is soft and slippery, the mechanical properties of asphalt concrete will be seriously weakened by adding a large amount of graphite, which is not good for the shear stress of asphalt concrete. However, the use of steel slag as aggregate can reduce the effect of the addition of conductive phase materials on pavement performance of asphalt concrete.

\section{Conductive mechanism of SGCAC}

The conductive mechanism of conductive composites is a hot topic in the research of conductive composites. At present, there are three popular conductive theories: conductive passage theory; tunnel effect theory and field emission theory. The conductive passage theory was first proposed by Kemp, and later modified by Parkinson, Hagood, Bulgin, and others. According to the conductive passage theory, the conductive paths can be formed when conductive particles are in contact with each other to form a chain network, or when the distance between conductive particles is less than $100 \mathrm{~nm}$. The more conductive particles per unit volume, the smaller the particle spacing, and the higher the conductivity of the composite. The theory of tunneling effect is based on quantum mechanics The theory holds that the conductivity of composite materials is not only conducted by the direct contact of conductive particles, when the conductive particles are close to a certain distance, there is also a certain tunnel formed by the electrons caused by thermal vibration between adjacent conductive particles to achieve directional migration and complete the conductive process[6]. According to the field emission theory proposed by Beek et al., the interface effect exists in the composite material. When the voltage increases to a specific value, the 
strong electric field between the conductive particles forms an emission electric field, which causes the electrons to pass through the insulator and generate a current effect.

The only conductive phase in ordinary graphite conductive asphalt concrete is graphite. Ding Qingjun through analysis and experimental verification, the conductive phenomenon of graphite conductive asphalt concrete is a combination of these three effects[1]. The base phase and graphite of SGCAC are both conductive phases, and the conductive network inside the material is rich, so its conductive mechanism is very complicated. Ao Zaoxin [7] obtained the schematic diagram of the conductive network of SGCAC through SMA gradation design, as shown in Figure 1.

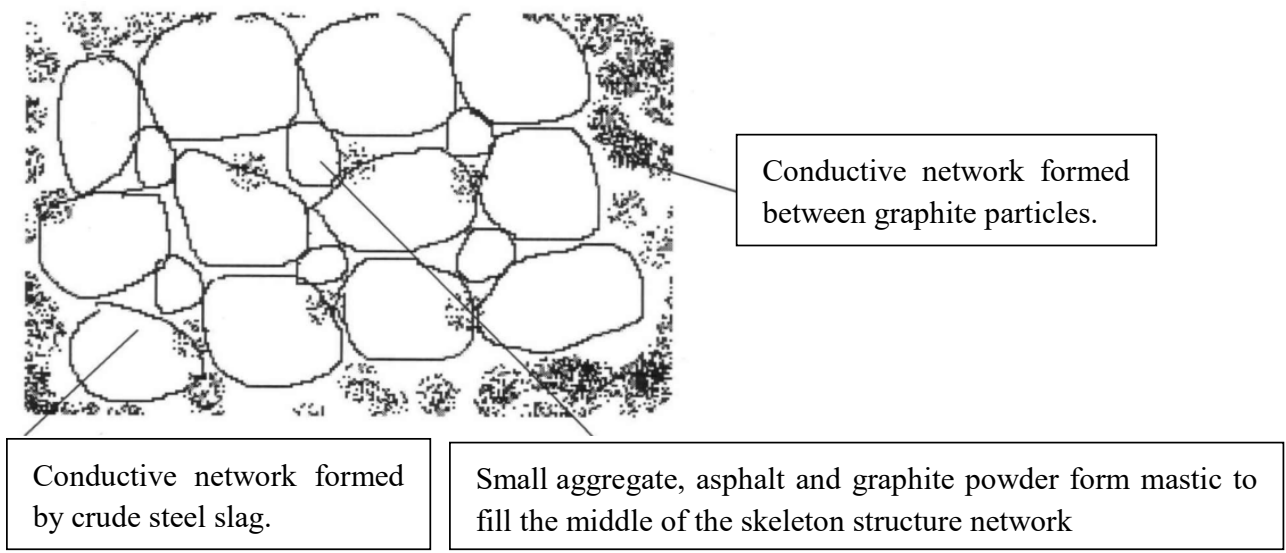

Fig. 1. Schematic of conductive network of SGCAC [7].

From figure 1, we can see that while the crude steel slag forms the skeleton structure, it contacts each other to form a conductive network of steel slag. Fine aggregate, asphalt, and graphite powder form mastic to fill the middle of the skeleton structure network, forming a more complete conductive network. In addition, the two-phase composite conductive material formed by asphalt and graphite is wrapped outside the aggregate, which is of great significance for improving the conductivity.

From this, we can conclude that there are three types of conductive chains in SGCAC: (1) steel slag conductive chains formed by overlapping steel slag and passing through each other; (2) graphite particle conductive chains formed by overlapping graphite particles with each other; (3)conductive chain formed by contact between steel slag and graphite. These three conductive chains are intertwined to form a complex three-dimensional conductive network.

\section{Electrical resistance stability of SGCAC}

Good electrical resistance stability is the basic requirement of conductive asphalt concrete as an electric heating material. The purpose of studying the electrical resistance stability of SGCAC is to maintain a good snow melting and ice melting effect even when external conditions change. [8]. The electrical resistance stability of SGCAC is mainly relate to the usage of time, external temperature, external pressure and chloride salt erosion. It is found that the external temperature has the greatest influence on the electrical electrical resistance stability of SGCAC.

There are two phenomena in the temperature stability of conductive asphalt concrete: one is the positive temperature coefficient effect of electrical resistance (PTC), and the 
other is the negative temperature coefficient effect of electrical resistance (NTC).PTC refers to that the resistivity of a material gradually increases with increasing temperature, and NTC refers to that the resistivity of a material decreases with increasing temperature.[9-10]. He Yongjia et al. measured the resistivity of SGCAC with different graphite content in the temperature range of $-30{ }^{\circ} \mathrm{C} \sim 20{ }^{\circ} \mathrm{C}$, as shown in Figure 2

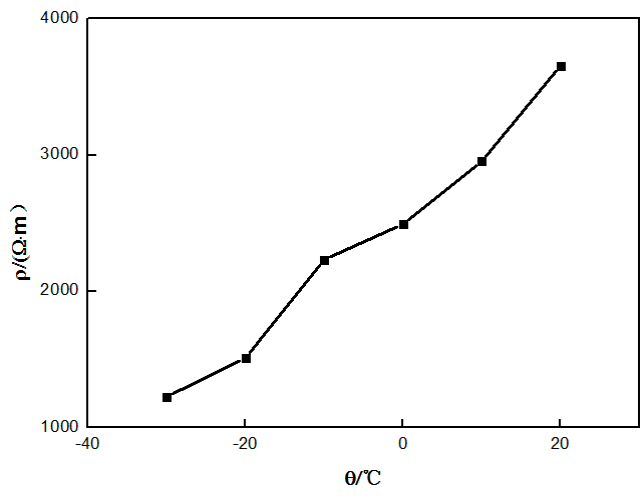

(a) $\varphi_{(\mathrm{C})=10 \%}$

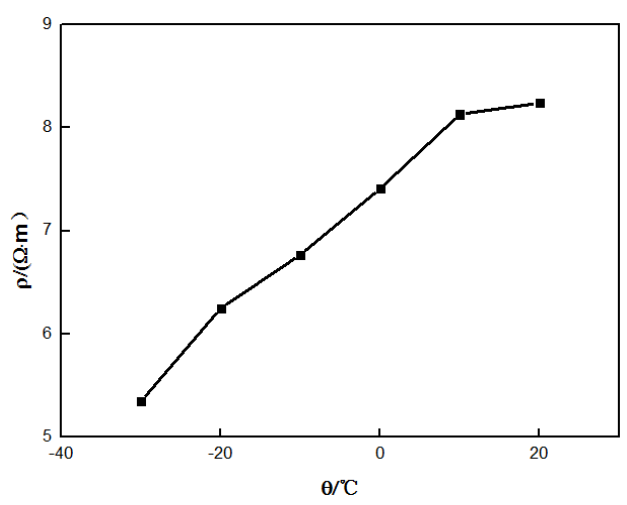

(c) $\varphi_{(\mathrm{C})=20 \%}$

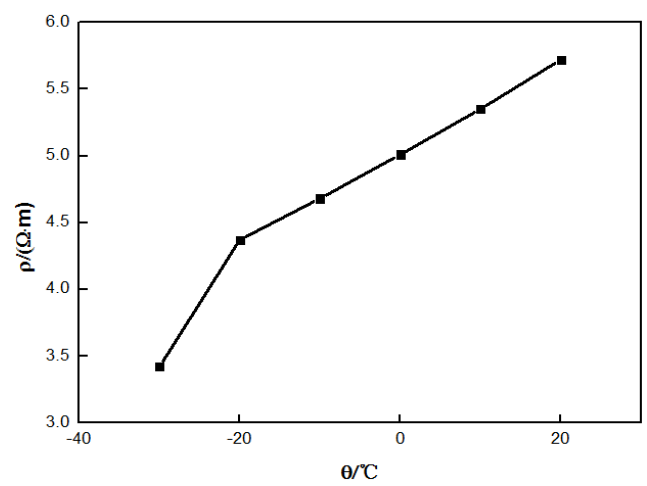

(e) $\varphi_{(\mathrm{C})=30 \%}$

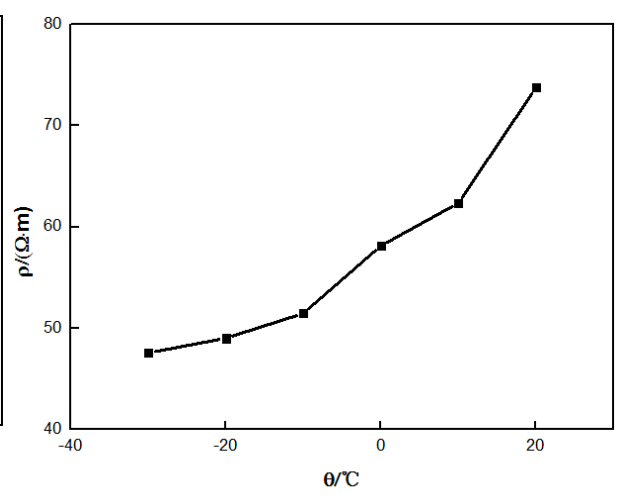

(b) $\varphi_{(\mathrm{C})=15 \%}$

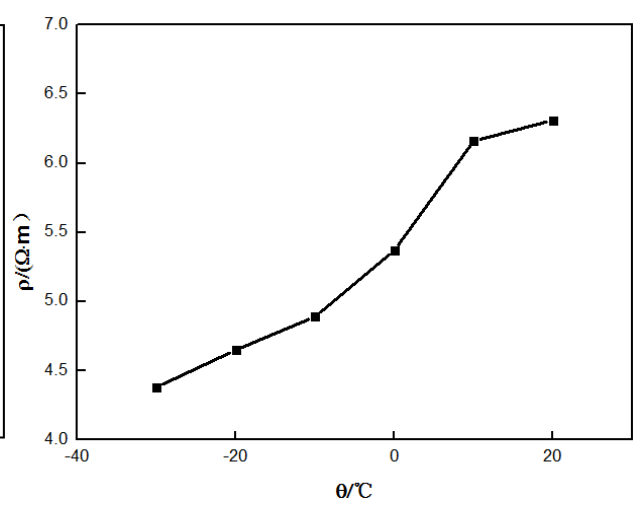

(d) $\varphi_{(\mathrm{C})=25 \%}$

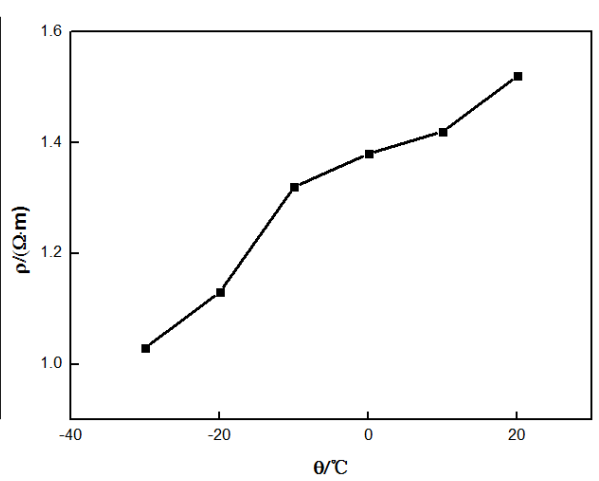

(f) $\varphi_{(\mathrm{C})=35 \%}$

Fig. 2. Electrical resistivity of SGCAC with different content of graphite at various temperatures [11]. 
From the figure, we can see that the SGCAC has obvious PTC effect, and its electrical resistivity increases with the gradual increase of temperature, indicating that the SGCAC has good temperature sensitivity. This is due to the different expansion coefficients of the steel slag particles and graphite conductive particles in the mixture, which causes their spatial displacements to change in the mixture, which is the result of the combined action of steel slag and graphite. From Figure 1, we can also see that the lower eletrical resistivity stage is the negative temperature stage, which provides favorable conditions for its use in melting snow and ice.

\section{Problems and prospects}

Many studies have shown that SGCAC has good electrical resistance stability [11-12], and the purpose of melting snow and icing can be achieved after being energized. At present, China is still in the theoretical research and laboratory stage for SGCAC, and has not yet been applied to engineering. This is mainly due to its relatively high cost and low electrothermal conversion rate.

Through the snow melting experiment, Chen Feng concluded that the heat generated by the electrothermal effect mainly has the following directions: the concrete heats itself; the snow is transferred to the top of the specimen for snow melting; and it is transferred to the baseplate and the air environment. In order to calculate the heat used for snow melting, he constructed a heat balance equation for SGCAC in the process snow melting:

$$
Q=C_{a} m_{a} \Delta T_{a}+C_{s} m_{s} \Delta T_{s}+\lambda_{s} m_{s}+Q_{1}
$$

Where $\mathrm{C}_{\mathrm{a}}, \mathrm{C}_{\mathrm{s}}$ - Specific heat capacity of base plate asphalt concrete and snow;

$\mathrm{M}_{\mathrm{a}}, \mathrm{m}_{\mathrm{s}}$ - base plate asphalt concrete and snow quality;

$\Delta T_{a}, \Delta T_{S}$ - the rising temperature of asphalt concrete and snow;

$\lambda_{s}$-the melting heat of snow;

$\mathrm{Q}$ - electric heat generated by conductive asphalt concrete;

$\mathrm{Q} 1$ - the environment loses heat

After the SGCAC was energized for 120 minutes, all the $5 \mathrm{~cm}$ of snow was melted, and the snow melting efficiency was calculated to be $32 \%$.

With the ever-increasing demands of modern traffic on road performance, improving and expanding road functions has become a hot research topic in the field of international roads. SGCAC is a new type of intelligent material, which can effectively remove snow on the road area in time and ensure road traffic safety. It will be widely used in the field of road construction and maintenance. In recent years, research on SGCAC has found that the following problems need to be further explored and resolved

\section{Conclusions}

1. Although the incorporation of graphite can reduce the electrical resistance value of steel slag graphite asphalt concrete, its high temperature stability and water stability are weakened. Therefore, it is necessary to find an appropriate graphite content in the future research process to ensure that the conductive performance of steel slag graphite asphalt concrete is improved without damaging its pavement performance.

2. It is harmful to the high temperature stability of SGCAC when heated by energizing. If 
it is repeatedly heated, it will accelerate the aging speed of asphalt and shorten the service life of the pavement

3. SGCAC has certain conductivity, but its electrothermal conversion efficiency is low. How to improve the electrothermal conversion efficiency of SGCAC under the precondition of ensuring pavement performance is a problem that needs to be further studied in the future.

\section{Reference}

1. D. Qingjun, W.U. Xuewei, A.O. Zaoxin, L. Xinquan, A.H. Shuguang, Highway 10, 167-172 (2008) DOI: 10.3969/j.issn.1000-033X.2010.11.024

2. C. Feng, W.S.-p., Z. Yuan, Construction machinery and Construction Technology 27(11), 51-54+74 (2010) DOI: 10.3969/j.issn.1000-033X.2010.11.024

3. M. Liantong, Preparation and Research of Conductive Asphalt Concrete (Wuhan University of Technology, 2004)

4. C. Kezheng, D. Fanglin, C. Zuolin, Z. Huakai, Synthetic rubber industry 6, 366-369 (2000) DOI: 10.3969/j.issn.1000-1255.2000.06.009

5. L. Changzheng, Y. Mingjin, Plastic 1, 32-34 (2000) DOI: 10.3969/j.issn.1001-9456.2000.01.008

6. K.P. Sau, D. Khastgir, T.K. Chaki, Die Angewandte Makromolekulare Chemie 258, 11-17 (1998)

7. A. Zaoxin, Research on Steel Slag Graphite Conductive Asphalt Concrete (Wuhan University of Technology, 2009)

8. S. Mingqing, Research on the Sensitivity and Application of Carbon Fiber Concrete and Plain Concrete (Wuhan University of Technology, 2002)

9. Y. Zhang, Journal of Applied Polymer Science 73, 489-494 (1999)

10. C.Y. Tuan, ACI Materials Journal 101(1), 65-71 (2004)

11. H. Yongjia, A. Zaoxin, L. Linnv, D. Qingjun, H. Shuguang, Journal of Beijing University of Technology 37(01), 80-84 (2011)

12. K. Krayushkina, O. Prentkovskis, A. Bieliatynskyi, R. Junevičius, Vilnius (Lietuva): Technika 27(2), 129-137 (2012) DOI: 10.3846/ 16484142.2012.690093 